Journal of the Magnetics Society of Japan Vol. 15 Supplement, No. S2 (1991)

(C) 1991 by The Magnetics Society of Japan

\title{
THE STUDY OF COCr/FeNi DOUBLE LAYERS FOR PERPENDICULAR MAGNETIC RECORDING
}

\author{
FU Yiliang * and XIA Zongning * *
}

* Dept. of Printing Technology,Beijing Institute of Printing, Beijing 102600,P. R. China. * * Dept. of Materials Science and Engineering,Tsinghua University, Beijing 100084,P. R. China.

\begin{abstract}
Both $\mathrm{FeNi}$ and $\mathrm{CoCr} / \mathrm{FeNi}$ films were prepared by a DC planar magnetron sputtering system. This paper describes the effects of sputtering conditions of FeNi films on the magnetic characteristics and structure of FeNi films and $\mathrm{CoCr} / \mathrm{FeNi}$ films. It was found that the FeNi film is sensitive to the deposition conditions ,the sputtering parameters have great influences on the (111) preferred orientation and the magnetic properties of the FCC FeNi films, satisfactory soft magnetic properties of $\mathrm{FeNi}$ films can be obtained by decreasing the argon pressure and the film thickness and/or raising the substrate temperature. The degree of C-axis preferred orientation of $\mathrm{CoCr}$ films deposited onto the FeNi films is strongly dependent on the degree of (111) plane orientation of $\mathrm{FeNi}$ films, this may be the result of the epitaxial growth of $\mathrm{CoCr}$ film with its (0002) plane parallel to the (111) plane of $\mathrm{FeNi}$ film. Some experimental results show the magnetic properties of $\mathrm{CoCr} / \mathrm{FeNi}$ double layers is related to that of the FeNi film. It is pointed out that a FeNi underlayer with satisfactory properties is the prerequisite condition for obtaining $\mathrm{CoCr} / \mathrm{FeNi}$ double layers with desired properties.
\end{abstract}

\section{INTRODUCTION}

Recently, much efforts have been made to study $\mathrm{CoCr} / \mathrm{FeNi}$ double layers as high density recording media ${ }^{[1]}$. The surface layer is $\mathrm{CoCr}$ film used as a perpendicular magnetization medium ${ }^{[2]}$, while the underlayer is FeNi film used to magnify the signal output level and to decrease the recording current. Many deposition parameters such as the argon pressure, the substrate bias voltage and the substrate temperature, etc, will affect the growth of FeNi films, and this will, in turn, influence the double layers.

This work presents the effects of varying the sputtering conditions of FeNi films on the magnetic characteristics and structures of both the FeNi underlayer and the $\mathrm{CoCr} / \mathrm{FeNi}$ double layers.

\section{EXPERIMENTAL}

A series of $\mathrm{FeNi}(20 \mathrm{at} \% \mathrm{Fe}-80 \mathrm{at} \% \mathrm{Ni})$ thin films with $500 \mathrm{~nm}$ thick was deposited by planar magnetron sputtering using high purity alloy targets, and $\mathrm{CoCr}(80$ at $\% \mathrm{Co}-20 \mathrm{at} \% \mathrm{Cr})$ thin films with $400 \mathrm{~nm}$ thick deposited onto FeNi films immediately. The deposition conditions for $\mathrm{CoCr}$ films were such that the argon pressure was kept at 0 . $46 \mathrm{~Pa}$ and deposition rate was as high as $200 \mathrm{~nm} /$ min. The substrate temperature can be set at any desired value by heating, ranging from 50 to about $400^{\circ} \mathrm{C}$. The substrate used for thin film deposition were well cleaned glass slides $(0.8 \mathrm{~mm}$ thick).

$\mathrm{X}$-ray diffraction method with $\mathrm{CuK} \alpha$ radiation (intensity versus $2 \theta$ scanning, rocking curve) were used to identify qualitatively the preferred orientation of the $\mathrm{FeNi}$ and $\mathrm{CoCr}$ films with respect to the specimen surface. A vibrating sample magnetometer (VSM) was used to measure the magnetic properties. 


\section{RESULTS}

1. The preffered orientation and the magnetic properties.

FIG. 1 gives $\mathrm{X}$-ray diffraction pattern of $\mathrm{CoCr} / \mathrm{FeNi}$ films deposited onto glass without heating substrate, two peaks are observed; one at $44.16^{\circ}$ corresponding to $\mathrm{FeNi}$ (111) diffraction, the other at $44.56^{\circ}$ corresponding to $\mathrm{CoCr}(0002)$ diffraction. The hysteresis loop ( $\mathrm{M}-\mathrm{H}$ curve) is shown in FIG. 2, which is typical for this $\mathrm{CoCr}$ film and indicates a low coercivity of about 2000 e in the normal direction of the film surface .

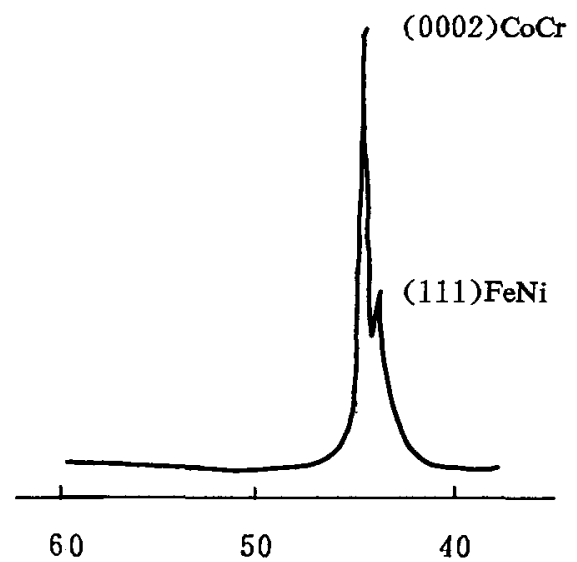

FIG. 1 X-Ray diffraction pattern of $\mathrm{CoCr} / \mathrm{FeNi}$ film

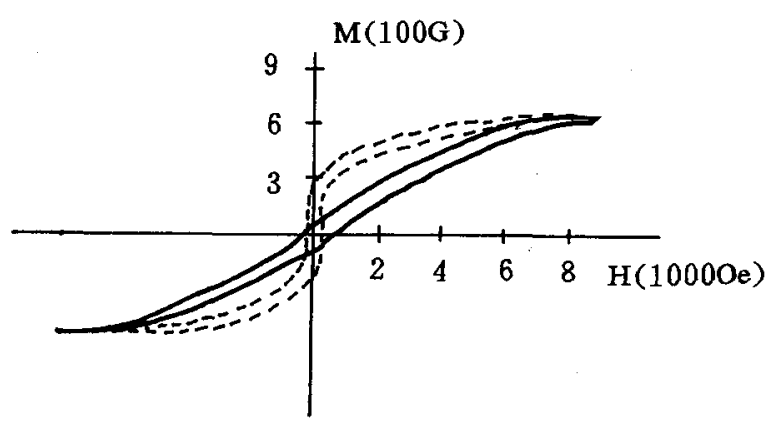

FIG. 2 M-H curve of $\mathrm{CoCr} / \mathrm{FeNi}$ film obtained with unheated substrate
2. The effects of the deposition conditions on the preferred orientation and properties of the thin films.

\section{a. Argon Pressure}

Table. 1 The influence of argon pressure on $\mathrm{FeNi}$ and $\mathrm{CoCr} / \mathrm{FeNi}$ films

\begin{tabular}{|c|rr|rrr|}
\hline Argon Pressure & \multicolumn{2}{|c|}{ FeNi films } & \multicolumn{3}{|c|}{$\mathrm{CoCr} / \mathrm{FeNi}$ films } \\
$(\mathrm{Pa})$ & $\Delta \theta_{50} \mathrm{Hc}_{/ /}(\mathrm{Oe})$ & $\Delta \theta_{50}$ & $\mathrm{Hc}_{\perp}(\mathrm{Oe})$ & $\mathrm{Hc}_{/ /}$ \\
\hline 0.26 & $8.8^{\circ}$ & 6.5 & $7.0^{\circ}$ & 127 & 36 \\
0.53 & $8.5^{\circ}$ & 6.4 & $7.2^{\circ}$ & 127 & 36 \\
1.06 & $9.4^{\circ}$ & 4.3 & $7.1^{\circ}$ & 91 & 36 \\
\hline
\end{tabular}

The effect of argon pressure is shown in Table. 1. From the table it is shown that with the increase of the argon pressure, the FeNi (111) plane becomes more and more scattered and both the in - plane coercivity $\mathrm{Hc}_{/ /}$of the FeNi film and the vertical coercivity $\mathrm{Hc}_{\perp}$ of the double layers decrease. However, the $(0002)$ preferred orientation of double layers is not significantly influenced by the argon pressure.

\section{b. The Thickness of FeNi Films}

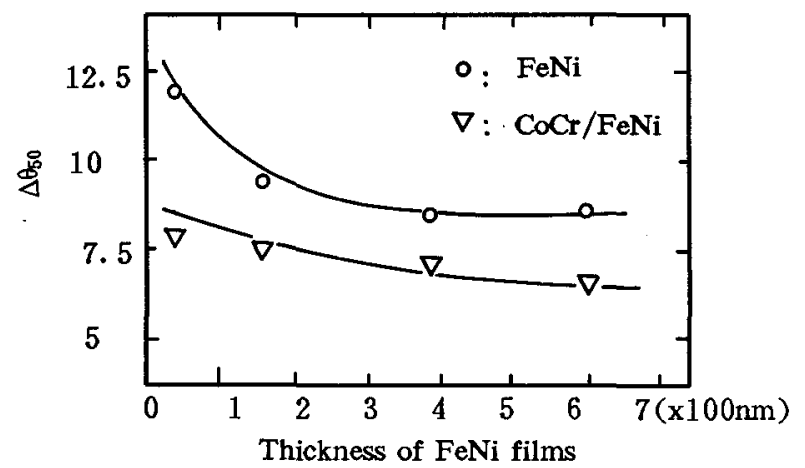

FIG. 3a $\Delta \theta_{50}$ of $\mathrm{FeNi}$ and $\mathrm{CoCr} / \mathrm{FeNi}$ versus the thickness of FeNi films. 


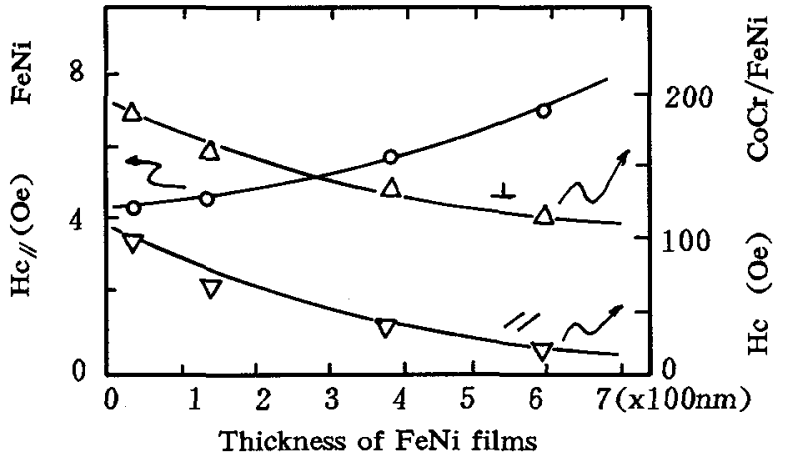

FIG. $3 \mathrm{~b} \mathrm{Hc}$ of $\mathrm{FeNi}$ and $\mathrm{CoCr} / \mathrm{FeNi}$ films versus the thickness of FeNi films.

The thickness of thin films can be controlled by adjusting sputtering time. There is a relationship between the thickness of $\mathrm{FeNi}$ film and the $\mathrm{FeNi}$ (111) and $\mathrm{CoCr}$ (0002) preferred orientations as shown in FIG. 3a, it can be seen that the increasing of the underlayers thickness improves the orientation of not only $\mathrm{FeNi}$ (111) plane but also $\mathrm{CoCr}$ (0002) plane. FIG. $3 \mathrm{~b}$ show the magnetic properties of both $\mathrm{FeNi}$ film and $\mathrm{CoCr} / \mathrm{FeNi}$ double layers as a function of the thickness of the underlayer FeNi. It can be seen from the figure that with the increasing of the underlayer thickness, the in-plane coercivity $\mathrm{Hc} / /$ of the FeNi films increases, while both the vertical and in-plane coercivity $\left(\mathrm{Hc}_{\perp}, \mathrm{Hc}_{/ /}\right)$of the double layers decrease. These results above show that the thickness of the underlayer must be controlled at some adequate value to ensure the films with satisfactory properties.

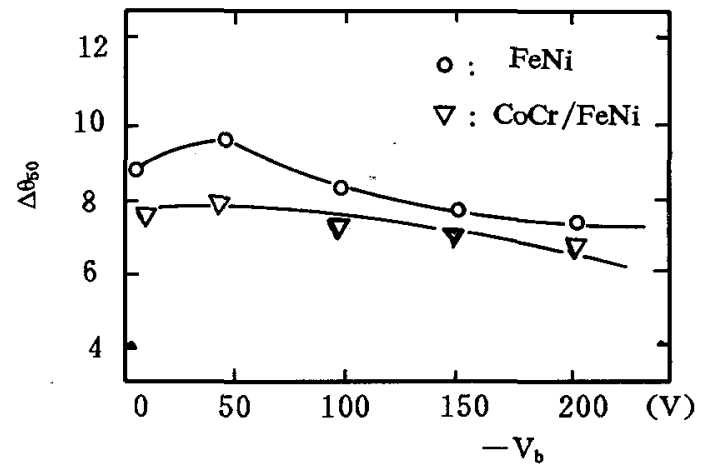

FIG. $4 a \Delta \theta_{50}$ of $\mathrm{FeNi}$ and $\mathrm{CoCr} / \mathrm{FeNi}$ films versus bias voltage given on deposition of FeNi films.

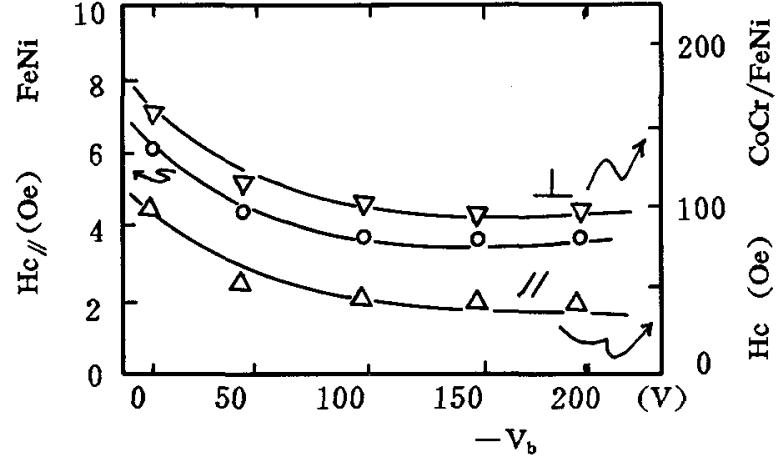

FIG. $4 b \mathrm{Hc}$ of $\mathrm{FeNi}$ and $\mathrm{CoCr} / \mathrm{FeNi}$ films versus bias voltage (on $\mathrm{FeNi}$ ).

\section{c. The Substrate Bias Voltage}

The bias voltage also has some effect on the structure of the films. As shown in FIG. 4a, with the decrease of bias voltage, the preferred orientation of $\mathrm{FeNi}$ films becomes more perfectly, while that of the double layer remains unchanged. FIG. $4 \mathrm{~b}$ shows the properties of sublayer and the double layers as a function of the bias voltage applied during the deposition of FeNi films. It can be seen from the figure that the coercivity deposited without the application of bias voltage is greater than those deposited under bias voltage of $-50 \mathrm{~V},-100 \mathrm{~V},-150 \mathrm{~V}$ and $200 \mathrm{~V}$ 's. In these voltage range, the $\mathrm{Hc}$ values are almost independent of the applied bias voltage.

\section{d. The Substrate Temperature}

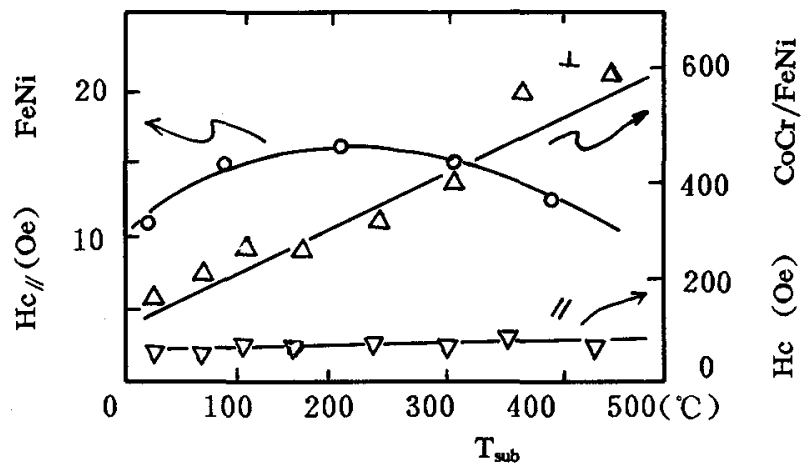

FIG. 5 The Coercivity of $\mathrm{FeNi}$ and $\mathrm{CoCr} / \mathrm{FeNi}$ film versus the substrate temperature. 
The films mentioned above were prepared with unheated substrate. In this case the $\mathrm{Hc}_{\perp}$ of the ciouble layers are quite low. To study the effect of the substrate temperature, some films were prepared at varying substrate temperature, FIG. 5 shows the dependence of the coercivity of the underlayer and the double layers on the substrate temperature. The figure indicates that raising the substrate temperature will lead to decrease of $\mathrm{Hc}_{/ /}$of underlayers and increase of the $\mathrm{Hc}_{\perp}$ of double layers, meanwhile, it has no effect on the $\mathrm{Hc}_{/ /}$of double layer. This result shows that the substrate temperature is an important factor for improving the magnetic characteristics of the underlayers and the double layers.

\section{DISCUSSIONS}

Based on the experimental results above, we can see that the (111) preferred orientation and the magnetic properties of the FeNi underlayers are sensitive to the deposition conditions. In particular, the underlayer has great influence on the $\mathrm{CoCr} / \mathrm{FeNi}$ double layers in the following aspects.

\section{a. The Epitaxial Growth Mechanism}

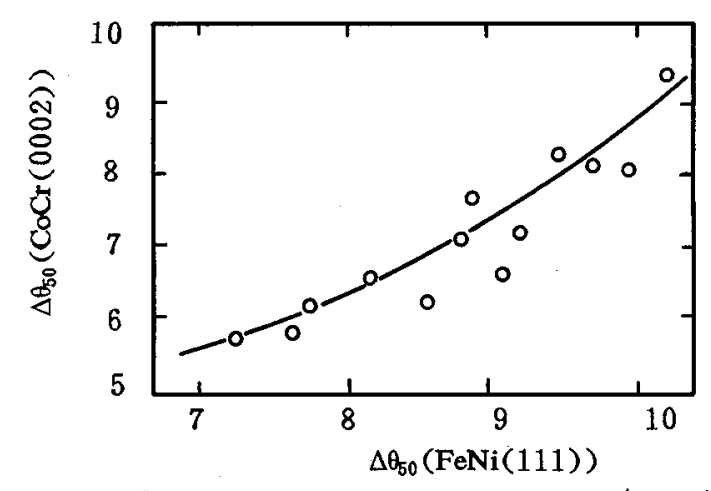

FIG. 6 The dependence of $\Delta \theta_{50}$ of $\mathrm{CoCr} / \mathrm{FeNi}(0002)$ on $\Delta \theta_{50}$ of $\mathrm{FeNi}(111)$.

FIG. 6 shows the relationship between the FeNi (111) preferred orientation and the $\mathrm{CoCr}(0002)$ preferred orientation. It is obvious from the figure that the $\mathrm{C}$-axis preferred orientation of the surface layer deposited onto the underlayers is highly affected by the (111) preferred orientation of the under- layers. The higher the degree of the FeNi (111) preferred orientation, the higher the degree of the $\mathrm{CoCr}$ (0002) preferred orientation. On the other hand, the interatomic distance in the $\mathrm{FeNi}$ (111) plane $(2.518 \AA)$ is approximately equal to that in the $\mathrm{CoCr}(0002)$ plane (2.523 $\AA$ ). From all those features above, the epitaxial growth mechanism-$\mathrm{CoCr}$ (0002) plane is epitaxial growth parallel to the FeNi (111) plane--can be confirmed. Some literatures ${ }^{[3][4][5]}$ also have given this result.

b. The Relationship Between Magnetic Properties.

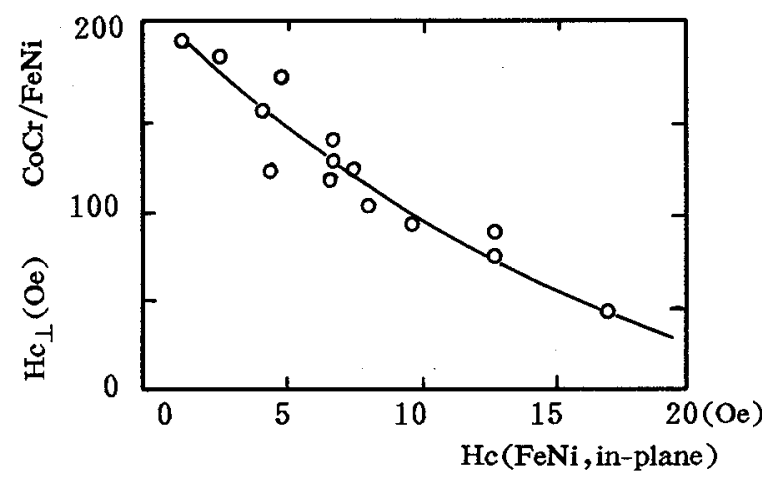

FIG. 7 The dependence of the coercivity of $\mathrm{CoCr} / \mathrm{FeNi}$ films $\left(\mathrm{Hc}_{\perp}\right)$ on the coercivity of $\mathrm{FeNi}(\mathrm{Hc} / /)$ films.

The magnetic properties of $\mathrm{FeNi}$ films have some effects on that of double layers as shown in FIG. 7. The figure shows that the $\mathrm{Hc}_{\perp}$ of $\mathrm{CoCr} /$ FeNi films decreases with the increasing of $\mathrm{Hc}_{\mathrm{c}}$ of FeNi films, this indicates that good soft properties of underlayer is closely related to the magnetic properties of double layers.

\section{CONCLUSIONS}

The results show that the underlayer $\mathrm{FeNi}$ films are sensitive to the deposition conditions. By decreasing the argon pressure and the film thickness and/or by raising the substrate temperature, one can obtain FeNi films with satisfactory soft magnetic properties. There is a close interrelationship between the $\mathrm{C}$-axis preferred orientation of the $\mathrm{CoCr}$ 
film and the (111) preferred orientation of the FeNi film. This may confirm the epitaxial growth mechanism of the $\mathrm{CoCr}$ film with its (0002) plane parallel to the (111) plane of the FeNi film. Since the magnetic properties of $\mathrm{CoCr} / \mathrm{FeNi}$ double layers is related to that of the FeNi films as found in our experiment, it is conclued that a $\mathrm{FeNi}$ underlayer with satisfactory soft properties is the prerequisite condition for obtaining $\mathrm{CoCr} / \mathrm{FeNi}$ double layers with desired properties.

\section{ACKNOWLEDGEMENT}

The authors express their appreciation to Dr.
GONG Shengkai and Dr. ZHOU Zhifeng for their useful discussions and help.

\section{REFERENCES}

[1]. S. IWASAKI and K. OUCHI, IEEE TRANS. MAGN. ;MAG-14,p849(1978).

[2]. S. IWASAKI, Y. NAKAMURA, and K. OUCHI, IEEE TRANS. MAGN. ;MAG-15, p1456 (1979).

[3]. G. CARGIN et al, IEEE TRANS. MAGN. ; MAG-15,p1821(1979).

[4]. Y. UESAKA et al, PROC. SENDAI SYMP. PER. REC. ;p169(1982).

[5]. R. SUGITA et al,PROC. JAP. ELECTR. COMM. CONF. ,p204(1984). 